\title{
Meeting Report
}

\section{All pathways to cancer apoptosis meeting in Thiruvananthapuram (India)}

\author{
K Mehta ${ }^{*, 1}$, V Gandhi ${ }^{1}$ and BB Aggarwal ${ }^{1}$ \\ 1 Department of Experimental Therapeutics, The University of Texas MD Anderson Cancer Center, Houston, TX 77030, USA \\ * Corresponding author: K Mehta, Department of Experimental Therapeutics, The University of Texas MD Anderson Cancer Center, Box 362,1515 Holcombe \\ Boulevard, Houston, TX 77030, USA. Tel: 713792 2649; Fax: 713745 4167; E-mail: kmehta@mdanderson.org
}

Cell Death and Differentiation (2006) 13, 2163-2164. doi:10.1038/sj.cdd.4401981; published online 2 June 2006

International Symposium on Translational Research: Apoptosis \& Cancer - Trivandrum (India) 18th to 21st December, 2005.

An extensive amount of research in the past three decades has revealed many pathways of cell death. Although 'apoptosis' is the term used for most types of cell death, the pathways that lead to it are not always the same. The list of agents that mediate apoptosis is constantly growing. Because cancer is a hyperproliferative disorder, apoptosis of cancer cells and not of normal cells is a critical issue. Both physiologic and pharmacologic inducers of apoptosis have been identified. At an international conference held in Kerala, India, scientists discussed how apoptosis occurs, what mediates it, what pharmacologic agents induce it, how it is genetically regulated, and its role in tumorigenesis and cancer.

It is said that multiple pathways can lead to enlightenment. What better place to discuss this than Thirvananthapuram (Trivandrum), Kerala. Created by Parsuram, the legendary Brahmin 'protector,' Kerala, the southernmost state in India, sits where the Indian Ocean and Arabian Sea meet, where Vasco de Gama landed in search of spices, where St. Thomas landed in search of enlightenment, where the world's second oldest mosque is located, and where one of the world's oldest synagogues is located.

The purpose of $3 \frac{1}{2}$-day conference that convened on December 18, 2005 was to bring together laboratory and clinical scientists to share current knowledge and future strategies for promoting the death of cancer cells. The participants included over 450 physicians and researchers from 16 different countries worldwide. The format of the meeting was daily major symposia, parallel minisymposia, and poster sessions.

Dr. Abdul Kalam, the President of India and an aerospace engineer, inaugurated the meeting and reminded the importance of stem cell cloning and nontoxic drugs in cancer treatment and the role of patient databases and genomics in cancer care.

The conference began with a keynote speech delivered by Dr. John Mendelsohn (Houston, TX, USA). He enlightened the audience by describing the journey that led to the discovery of Erbitux, a monoclonal antibody against epidermal growth factor receptor (EGFR). Major highlight of Dr.
Mendelsohn's talk was that although this antibody induces apoptosis of EGFR-overexpressing tumor cells in the laboratory, its effect on tumor cells in patients was not mediated through EGFR but through antibody-dependent cell-mediated cytotoxicity. Erbitux when combined with chemotherapeutic agents is effective in some patients with colorectal cancer. Another interesting revelation was that this antibody induces antitumor response in patients that is unrelated to the level of EGFR expression in tumor cells.

In a plenary lecture, Dr. Bharat Aggarwal (Houston, TX, USA), explained the connection between inflammation and cancer. A common molecule in these two processes is the tumor necrosis factor (TNF). He pointed out that natural products could serve as good drugs for treatment of both inflammation and cancer. One such product is curcumin, which exhibits a blocking effect on the TNF. Curcumin is being tested in early clinical trials in patients with multiple myeloma, breast cancer, and pancreatic cancer. Dr. S Krishna (Banglore, India) delineated the Notch oncogenic signaling pathway in human epithelial neoplasms. His group has identified deregulated Notch signaling in cervical cancer. The midmorning session began with a speech by Dr. Premkumar Reddy (Philadelphia, PA, USA), who discussed the human kinome. Eucaryotic and atypical protein kinases of the human kinome constitute more than 500 proteins mostly belonging to tyrosine kinases or serine/threonine kinases. Many of these kinases are either mutated, constitutively active, or overexpressed in human cancers. The best example of such an agent is imatinib, the inhibitor of Bcr-Abl tyrosine kinase, he pointed out.

Dr. Raj Puri (Bethesda, MD, USA) discussed early laboratory, preclinical, and animal model studies that were conducted with an immunotoxin composed of $\mathrm{IL}-13$ and a mutated form of Pseudomonas exotoxin. These preclinical results were translated into phase $\mathrm{I} / \mathrm{II}$ investigations in patients with solid tumors. Dr. Kapil Mehta (Houston, TX, USA) discussed the implications of elevated tissue transglutaminase expression in the development of drug resistance and metastatic phenotypes in tumor cells. 
The major points of the afternoon symposium were inflammation, NF- $\kappa \mathrm{B}$, and cancer. At a plenary session, Dr. Michael Karin (La Jolla, CA, USA) illustrated how the I kappa B kinase (IKK) complex provides a link between inflammation and cancer. His data suggested that the IKK/NF- $\kappa$ B pathway is not only involved in suppression of apoptosis in advanced cancers but that its inactivation could attenuate formation of inflammation-associated tumors. Dr. Vinay Tergaonkar (Singapore) described a genomic approach to identify molecules and proteins that regulate $\mathrm{NF}-\kappa \mathrm{B}$ function and TRF-IP/Rap-1 was one such protein identified by this approach. Dr. Ajit Verma (Madison, WI, USA) presented evidence that transgenic mice, which overexpress $\mathrm{PKC} \varepsilon$ in the epidermis, were highly sensitive to ultraviolet (UV) radiationinduced squamous cell carcinoma. PKC $\varepsilon$-mediated loss of FADD expression inhibited UV radiation-induced activation of both extrinsic and intrinsic apoptotic pathways. Dr. Bhudev Das (Gurgaon, India) reported on constitutive expression of $\mathrm{NF}-\kappa \mathrm{B}$ in cervical carcinomas.

At three parallel minisymposia, discussion focused on antiand pro-apoptotic pathways in cancer, identification of and preclinical studies of natural products for treatment of cancer, and signal transduction pathways.

Next day morning started with a plenary talk by Dr. S Nagata (Osaka, Japan), who discussed the mechanism by which debris generated by dead cells is cleared. He explained that cells that lack CAD do not undergo DNA fragmentation. Instead, their DNA is degraded by DNAse II. Dr. Nagata indicated that DNAse II deficiency in embryonic mice is lethal. Dr. V Gandhi (Houston, TX, USA) discussed preclinical and clinical studies with nelarabine, a nucleoside analog. Nelarabine was recently approved by the US Food and Drug Administration for use in treating relapsed $\mathrm{T}$-cell leukemias and lymphomas.

Dr. Vishva Dixit (San Francisco, CA, USA), in a plenary lecture, described the role of the constitutively photomorphogenic 1 (COP1) and de-Etiolated (D ET) phenotypes in repressing plant photomorphogenesis, the light-mediated program of plant development.

Dr. Michael Andreef (Houston, TX, USA) discussed the role of mesenchymal stem cells (MSC) for tumor stroma and targeted delivery vehicles for anticancer agents. His data suggest that systemically administered gene-modified MSC can inhibit the growth of metastatic breast cancer, melanoma, ovarian cancer, and myeloid leukemia and that chromosomal abberations are closely connected with clinical response in acute mylogenous leukemia. Dr. Lalitha Nagarajan (Houston, TX, USA) discussed the role of tumor suppressor gene SSBP2. The loss of SSBP2 in conjunction with loss of TP53 confers a block in differentiation and a survival advantage to blasts.

Dr. K Somasundaram (Banglore, India) discussed the role of Notch-1 in chemoresistance; overexpression of the intracellular domain of Notch-1 induces resistance to chemotherapy through inhibition of p53 phosphorylation. Dr. Atul Bedi (Baltimore, MD, USA) discussed the resistance of cancer cells to adoptive immunotherapy via $X$-linked inhibitor of apoptosis (XIAP) protein expression and coexisting defects in mitochondrial death signaling. Finally, Dr. K Ramadas (Trivandrum, India) provided an update on the status of oral cancer screening efforts in India. Oral cancer is the most common cancer in that country.

On the final day of the conference, Dr. Jorge Moscot (Madrid, Spain) discussed the interplay between protein kinase $\mathrm{C}$, Janus kinase $1, \mathrm{NF}-\kappa \mathrm{B}$, and the Par4 signaling pathways. In $\mathrm{Par}^{-1-}$, female mice developed endometrial carcinomas whereas male mice developed prostatic intraepithelial neoplasias. Moreover, Par4-null tissues and cells showed increased levels of the anti-apoptotic protein XIAP as a result of NF- $\kappa$ B activation. Dr. Gopal Kundu (Pune, India) reported the osteopontin-induced $\mathrm{PI3}-\mathrm{K}$-dependent activation of NF- $\kappa \mathrm{B}$ and secretion of urokinase plasminogen activator (UPA). Osteopontin also regulated the transactivation of EGFR in a c-Src-dependent manner resulting in AP-1 activation, UPA secretion, and cell motility through mitogenactivated protein kinase (MAPK) signaling pathways. According to Dr. D Karunagaran, (Thirvananthapuram, India), constitutive activation of $\mathrm{NF}-\kappa \mathrm{B}$ protects cancer cells from curcumin-induced apoptosis. The Bcl-xl protein and the $\mathrm{Ku} 70$ enzyme also protect cells from curcumin-induced apoptosis by inhibiting the release of cytochrome $c$, Smac, and apoptosis-inducing factor (AIF) protein and activating caspase-9, -8, and -3. Dr. Promila Singh (Galveston, TX, USA) observed that presence of progastrin and insulin-like growth factor (IGF)-Il could alleviate curcumin-induced apoptosis of colorectal cancer cells. Indeed, progastrin and IGF-II are constitutively expressed in most colon cancers. Dr. Jasti Rao (Peoria, IL, USA) presented evidence that the use of bicistronic plasmid constructs to induce RNA interferences targeted against two different proteases (e.g., UPAR and cathepsin B or UPAR and MMP-9) is highly effective in inhibiting tumor growth both in vitro and in xenograft models. Finally, Dr. D Raghunandharao (Madison, WI, USA) presented the results of a phase II clinical study of talactoferrin (TLF) alfa, an oral immunomodulatory protein, in 110 patients with advanced non-small-cell lung cancer. There was a significant difference in survival of patients with and without a partial response to carboplatin/paclitaxel plus TLF $(P<0.01)$, suggesting a strong association between confirmed RR and survival.

Overall, the meeting was a great success. Dr. Mendelsohn concluded the conference by summarizing the highlights.

\section{Acknowledgements}

We thank the local organizers (Drs. O Oommen, MR Pillai, and PR Sudhakaran, our speakers and attendees for making the conference a successful event, the Office of International Affairs (National Cancer Institute, USA), the Kerala State Council for Science and Technology, the Indian Council of Medical Research, the Council of Scientific and Industrial Research (India), the Department of Science and Technology (India), the Department of Biotechnology (India), and several other sponsors. We apologize to those speakers whose work is not discussed owing to space limitations. We thank Manuel Gonzales for editorial help. 